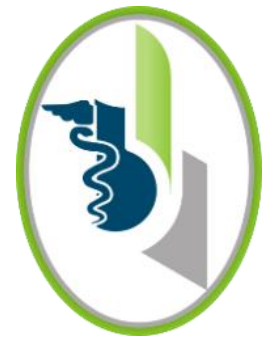

ACCESO $\odot$ ABIERTO

Para citaciones: Osorio, C. Ballestas, J., Martinez, J., Acevedo, Z., Barrios, D., Herrera, F., Redondo, C., Redondo, K. (2019). Características

anatomopatológicas del carcinoma papilar de tiroides en especimenes con y sin tiroiditis linfocitica cronica Revista Ciencias Biomédicas, 8(2), 32-39.

Recibido: 6 de marzo de 2019 Aprobado: 30 de abril de 2019

Autor de correspondencia: Carlos Gustavo Osorio Covo cosorioc@gruposcalpellum.com

Editor: Inés Benedetti. Universidad de Cartagena-Colombia.

Copyright: (C) 2019. Osorio, C. Ballestas, J., Martinez, J., Acevedo, Z., Barrios, D., Herrera, F., Redondo, C., Redondo, K. Este es un artículo de acceso abierto, distribuido bajo los términos de la licencia https://creativecommons.org/licenses/bync-sa/4.0/ la cual permite el uso sin restricciones, distribución y reproducción en cualquier medio, siempre y cuando el original, el autor y la fuente sean acreditados.

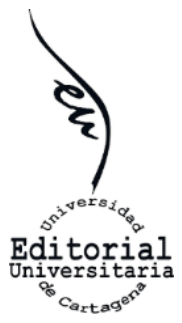

\section{Características anatomopatológicas del carcinoma papilar de tiroides en especimenes con y sin tiroiditis linfocitica cronica}

\author{
Anatomopathological characteristics of papillary thyroid \\ carcinoma in specimens with and without chronic lymphocytic \\ thyroiditis.
}

Carlos Osorio Covo ${ }^{1}$, Jorge Ballestas Barrera ${ }^{1}$, Jorge Martínez Castro ${ }^{1}$, Zully Acevedo Meza ${ }^{2}$, Diego Barrios Castellar ${ }^{3}$, Francisco Herrera Sáenz ${ }^{1,4}$, Cesar Redondo Bermúdez ${ }^{2,5}$, Katherine Redondo De Oro ${ }^{2,5}$

${ }^{1}$ Sección de Cirugia General. Grupo de Investigación Scalpellum. Facultad de Medicina, Universidad de Cartagena, Cartagena, Colombia.

${ }^{2}$ Sección de Patología. Grupo de Investigación Scalpellum. Facultad de Medicina, Universidad de Cartagena, Cartagena, Colombia.

${ }^{3}$ Semillero de Investigación Scalpellum. Facultad de Medicina, Universidad de Cartagena, Cartagena, Colombia.

${ }^{4}$ Servicio de Cirugia General, E.S.E. Hospital Universitario del Caribe, Cartagena, Colombia.

${ }^{5}$ Servicio de Anatomía Patológica, E.S.E. Hospital Universitario del Caribe, Cartagena, Colombia

\section{RESUMEN}

Introducción: La hipótesis que sugiere asociación entre el Carcinoma Papilar de Tiroides (CPT) y la Tiroiditis Linfocítica Crónica (TLC) sigue generando debate en la actualidad. Estudios retrospectivos sugieren que el comportamiento biológico del CPT asociado a TLC es menos agresivo en comparación con el CPT no asociado a TLC.

Objetivo: Comparar las características anatomopatológicas del CPT en pacientes con y $\sin$ TLC en la E.S.E. Hospital Universitario del Caribe durante el periodo 2008 $-2018$.

Métodos: Se realizó un estudio analítico, de medición transversal y temporalidad retrospectiva. Se documentó el género, la edad, el diagnóstico de TLC y CPT en especímenes quirúrgicos productos de tiroidectomías en la E.S.E. Hospital Universitario del Caribe durante el periodo 2008 - 2018. Se comparó el subtipo histológico, el tamaño tumoral, la frecuencia de angioinvasión, la frecuencia de extensión tumoral extratiroidea, la focalidad, la frecuencia de metástasis cervicales y la frecuencia de metástasis a distancia en pacientes con y sin TLC.

Resultados: La población de estudio estuvo conformada por 177 pacientes, 151 mujeres $(85.3 \%)$ y 26 hombres $(14.7 \%)$, con una edad promedio de $45.8 \pm 14.9$ años. La frecuencia de pacientes con coexistencia de CPT y TLC fue de $23.7 \%$, correspondiente a 42 pacientes. La probabilidad de identificar un subtipo histológico (OR 0.602, IC95\% 0.291-1.223), un tamaño tumoral especifico (OR 0.596, IC95\% 0.280-1.270), angioinvasión (OR 0.625 IC95\% 0.222-1.756), extensión tumoral extratiroidea (OR 0.559 IC95\% 0.216-1.450), una focalidad tumoral especifica (OR 0.976, IC95\% 0.444-2.143) y compromiso ganglionar (OR 1.281, IC95\% 0.6002.736), no estuvo asociada con la presencia de TLC en el espécimen quirúrgico. 
Conclusiones: En nuestro estudio de investigación las características anatomopatológicas del CPT fueron similares en el grupo de pacientes con TLC y el grupo de pacientes sin TLC. Debido a la retrospectividad del presente estudio, recomendamos la realización de estudios prospectivos en los cuales se definan las características anatomopatológicas del CPT con criterios actualizados y universales.

Palabras Clave: Tiroiditis Linfocítica Crónica; Carcinoma Papilar de Tiroides; Características Anatomopatológicas.

\section{ABSTRACT}

Introduction: The hypothesis that suggests association between Papillary Thyroid Carcinoma (PTC) and Chronic Lymphocytic Thyroiditis (CLT) continues to generate debate at present. Retrospective studies suggest that the biological behavior of PTC associated with CLT is less aggressive compared to PTC not associated with CLT.

Objective: To compare the anatomopathological characteristics of PTC in patients with and without CLT in the E.S.E. University Hospital of the Caribbean during the period $2008-2018$.

Methods: An analytical, transversal measurement and retrospective temporality study was carried out. We documented gender, age, diagnosis of CLT and PTC in surgical specimens of thyroidectomy products in the E.S.E. University Hospital of the Caribbean during the period 2008 - 2018. The histological subtype, tumor size, frequency of angioinvasion, frequency of extrathyroidal tumor extension, focality, frequency of cervical metastasis and the frequency of distant metastasis were compared between patients with and without CLT.

Results: Were included 177 patients, 151 women (85.3\%) and 26 men (14.7\%), with an average age of $45.8 \pm 14.9$ years. The frequency of patients with coexistence of PTC and CLT was $23.7 \%$, corresponding to 42 patients. The probability of identifying a histological subtype (OR 0.602, IC95\% 0.291-1.223), a specific tumor size (OR 0.596, IC95\% 0.280-1.270), angioinvasion (OR 0.625 IC95\% 0.2221.756), extrathyroidal tumor extension (OR 0.559 IC95\% 0.216-1.450), a specific tumor focality (OR $0.976,95 \%$ CI $0.444-2.143$ ) and lymph node involvement (OR 1281, IC95\% 0.600-2736), was not associated with the presence of CLT in the surgical specimen.

Conclusion: In our study, the anatomopathological characteristics of the PTC were similar in the group of patients with CLT and the patient group without CLT. Due to the retrospective nature of the present study, we recommend carrying out prospective studies in which the anatomopathological characteristics of the PTC are defined with updated and universal criteria.

Key Words: Chronic Lymphocytic Thyroiditis; Papillary Thyroid Carcinoma. 


\section{INTRODUCCIÓN}

La Tiroiditis Linfocítica Crónica (TLC), también denominada "Tiroiditis de Hashimoto", es la causa más frecuente de hipotiroidismo en las zonas del mundo con adecuada suplencia de yodo (1). La edad de mayor incidencia se ubica entre la quinta y sexta década de la vida, es considerada una enfermedad propia del género femenino, con una relación mujer: hombre de aproximadamente 7: 1 (2). El nombre de la enfermedad se adjudicó en honor al cirujano japonés Hakaru Hashimoto, quien en 1912 describió cuatro mujeres con bocio en cuyo estudio histológico observó una aparente transformación del parénquima tiroideo en tejido linfoide, describiendo el esbozo de la enfermedad bajo el nombre de "struma lymphomatosa" (3).

En el año 2018 el carcinoma papilar (CPT) representó el 90\% de las neoplasias malignas de la glándula tiroides, el 95\% de todas las neoplasias malignas derivadas de órganos endocrinos y el 3.1\% del total de neoplasias malignas (4). En Estados Unidos entre el año 1969 y 2018, la incidencia de CPT se triplicó, aumentando de 4.8 a 14.5 casos por cada 100.000 habitantes/año (5), alcanzando una frecuencia del $1.5 \%$ y del $4.6 \%$ entre los tumores malignos en hombres y mujeres, respectivamente, y para el caso de la mujer, ocupó el quinto lugar en incidencia, superado por el cáncer de mama (30\%), el cáncer de pulmón (13\%), el cáncer colorrectal (7\%) y el cáncer del cuerpo uterino $(7 \%)(4,6)$.

El primer informe encontrado en la literatura, en el cual se reporta la frecuencia de CPT en pacientes con TLC fue publicado en 1952 por Lindsay y Cols, quienes documentaron una prevalencia del $21 \%$ (7). Sin embargo, la hipótesis que supone una mayor prevalencia de CPT en pacientes con TLC fue evaluada por primera vez por Dailey y Cols en 1955 (8). Desde entonces la posible asociación entre ambas patologías ha sido documentada en múltiples series. Un ámbito muy poco estudiado en torno la asociación entre el CPT y la TLC consiste en el comportamiento biológico del tumor en el contexto de una TLC. Un metaanálisis desarrollado por Moon incluyó 71 estudios con un total de 44,034 pacientes con CPT, y comparó las características anatomopatológicas de este tumor en pacientes con y sin hallazgos compatibles con TLC en los especímenes quirúrgicos. Sus resultados arrojaron que en pacientes con TLC el CPT parecía mostrar características anatomopatológicas más favorables en comparación con aquellos pacientes con CPT sin TLC (9). El objetivo del estudio consistió en comparar las características anatomopatológicas del CPT en pacientes con y sin TLC en la E.S.E. Hospital Universitario del Caribe durante el periodo 2008 - 2018.

\section{MÉTODOS}

Se realizó un estudio analítico, de medición transversal y de temporalidad retrospectiva (10). Se revisó el historial clínico de los 1874 pacientes manejados en el Servicio de Cirugía de Cabeza y Cuello de la E.S.E. Hospital Universitario del Caribe, de Cartagena, Colombia, entre enero de 2008 y diciembre de 2018. Se contó con el aval del Departamento de Investigaciones de la Facultad de Medicina de la Universidad de Cartagena y el Comité de Ética de la E.S.E. Hospital Universitario del Caribe.

La población de estudio estuvo conformada por 177 pacientes con diagnóstico de CPT que contaban con el estudio anatomopatológico del espécimen quirúrgico. Se revisó la descripción macroscópica y el diagnóstico anatomopatológico en los especímenes quirúrgicos de cada paciente. El diagnóstico histológico de CPT se realizó con base en los criterios descritos en la cuarta edición de la Clasificación Internacional de los Tumores de Órganos Endocrinos, propuesta por la Organización Mundial de la Salud (11). El diagnostico de TLC se realizó en base al hallazgo de un reemplazo difuso del parénquima tiroideo por un infiltrado mononuclear mediado por linfocitos y plasmocitos, agrupados en folículos con centros germinales, asociado a un variable grado de atrofia folicular con o sin células de Hürthle (12). La neoplasia folicular encapsulada no invasiva con características nucleares similares al carcinoma papilar (NIFTP) fue considerada una entidad diferente al CPT (13). 
El conjunto de definiciones correspondientes a las características anatomopatológicas del CPT fueron realizadas de acuerdo con los parámetros estipulados por el Instituto Nacional de Cancerología, Bogotá, Colombia (14).

Las variables cualitativas se presentaron en forma de tablas de frecuencia. Las variables cuantitativas se presentaron como medidas de tendencia central según el cumplimiento de los criterios de normalidad (15). El diagnóstico de TLC fue operacionalizado como una variable cualitativa, nominal y dicotómica; se categorizó en dos grupos: pacientes con TLC y sin TLC. Se documentaron las siguientes características anatomopatológicas: el subtipo histológico del CPT fue operacionalizado como una variable cualitativa, nominal y dicotómica; se categorizó en dos grupos: variante clásica y variante folicular. Los casos correspondientes a variantes agresivas como la variante columnar, la variante de células altas, y la variante esclerosante difusa, no fueron incluidos en el análisis estadístico.

El tamaño tumoral fue operacionalizado como una variable cualitativa, nominal y dicotómica; se categorizó en dos grupos: tumores con tamaño igual o menor a $10 \mathrm{~mm}$ y tumores con tamaño mayor a 10 $\mathrm{mm}$. La angioinvasión se operacionalizó como una variable cualitativa, nominal y dicotómica; se categorizó en dos grupos: angioinvasión presente y angioinvasión ausente. La extensión tumoral extratiroidea se operacionalizó como una variable cualitativa, nominal y politómica; se categorizó en dos grupos: extensión tumoral extratiroidea presente y extensión tumoral extratiroidea ausente. La focalidad se operacionalizó como una variable cualitativa, nominal y politómica; se categorizó en dos grupos: tumor unifocal y tumor multifocal. El compromiso ganglionar cervical se operacionalizó como una variable cualitativa, nominal y politómica; se categorizó en dos grupos: compromiso ganglionar presente y compromiso ganglionar ausente. La metástasis a distancia se operacionalizó como una variable cualitativa, nominal y politómica; se categorizó en dos grupos: metástasis a distancia presente y metástasis a distancia ausente.

\section{Análisis estadístico}

Se calcularon la razón de prevalencias (RP) y la razón de momios (OR) (16). Se calculó la significancia estadística de dicha asociación mediante la prueba Chi-Cuadrado de Pearson (17). Los valores de $p$ menores a 0.05 fueron considerados estadísticamente significativos. El análisis de los datos fue realizado con el paquete estadístico IBM SPSS Statistics 25.0.

\section{RESULTADOS}

La población de estudio estuvo conformada por 177 pacientes, 151 mujeres (85.3\%) y 26 hombres (14.7\%), con una edad promedio de $45.8 \pm 14.9$ años. La frecuencia de pacientes con coexistencia de CPT y TLC fue de $23.7 \%$, correspondiente a 42 pacientes. La comparación entre las características anatomopatológicas del CPT en pacientes con y sin TLC se resume en la tabla 1.

Se encontraron frecuencias de $66.1 \%$ y $33.9 \%$ para los subtipos clásico y folicular en la población de estudio, respectivamente. La prevalencia del subtipo clásico $(57.1 \%$ vs $68.8 \%)$ y la prevalencia del subtipo folicular (42.9 vs. $31.2 \%)$ fueron estadísticamente homogéneas en el grupo de pacientes con TLC y sin TLC (RP 0.68, IC95\% 0.481.23). La probabilidad de identificar un subtipo histológico específico no estuvo asociada con la presencia de TLC en el espécimen quirúrgico (OR 0.602, IC95\% 0.291-1.223). Esta asociación no fue estadísticamente significativa, con un valor de $\mathrm{p}=$ 0.160 .

Se encontraron frecuencias de $25.4 \%$ y $74.6 \%$ para tumores con tamaño $\leq 10 \mathrm{~mm} \mathrm{y}>10 \mathrm{~mm}$ en la población de estudio, respectivamente. La prevalencia de tumores con tamaño $\leq 10 \mathrm{~mm}(31.1 \%$ vs. $22.9 \%$ ) y la prevalencia de tumores con tamaño $>10 \mathrm{~mm}(66.7 \%$ vs. $77.1 \%)$ fueron estadísticamente homogéneas en el grupo de pacientes con TLC y sin TLC (RP 1.47, IC95\% 0.85-2.53). La probabilidad de identificar un tamaño tumoral específico no estuvo asociada con la presencia de TLC en el espécimen quirúrgico (OR 0.596, IC95\% 0.2801.270). Esta asociación no fue estadísticamente significativa, con un valor de $\mathrm{p}=0.178$. 


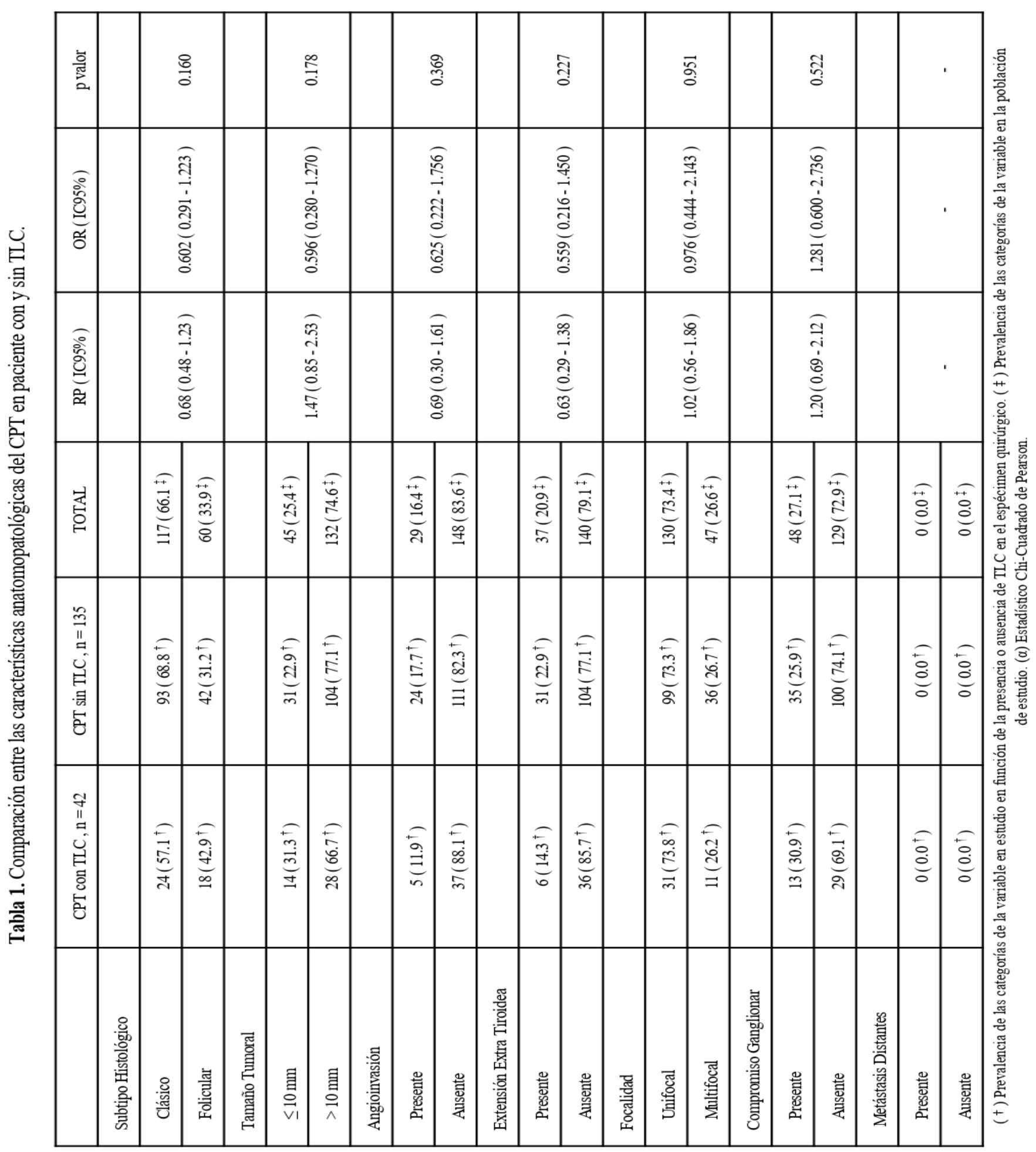

Se encontró una frecuencia de angioinvasión correspondiente al $16.4 \%$. La prevalencia de angioinvasión fue estadísticamente homogénea en el grupo de pacientes con TLC y sin TLC (11.9\% vs.
17.7\%, RP 0.69, IC95\% 0.30-1.61). La probabilidad de identificar angioinvasión no estuvo asociada con la presencia de TLC en el espécimen quirúrgico (OR 0.625 IC95\% 0.222-1.756). Esta asociación no fue 
estadísticamente significativa, con un valor de $\mathrm{p}=$ 0.369 .

Se encontró una frecuencia de extensión tumoral extratiroidea correspondiente al $20.9 \%$. La prevalencia de extensión tumoral extratiroidea fue estadísticamente homogénea en el grupo de pacientes con TLC y sin TLC (14.3\% vs. $22.9 \%$, RP 0.63, IC95\% 0.29-1.38). La probabilidad de identificar extensión tumoral extratiroidea no estuvo asociada con la presencia de TLC en el espécimen quirúrgico (OR 0.559 IC95\% 0.216-1.450). Esta asociación no fue estadísticamente significativa, con un valor de $\mathrm{p}=0.227$.

Se encontraron frecuencias de $73.4 \%$ y $26.6 \%$ para tumores unifocales y multifocales en la población de estudio, respectivamente. La prevalencia de tumores unifocales $(73.8 \%$ vs $73.3 \%$ ) y la prevalencia de tumores multifocales (26.2 vs. 26.7\%) fueron estadísticamente homogéneas en el grupo de pacientes con TLC y sin TLC (RP 1.02, IC95\% 0.561.86). La probabilidad de identificar un tipo de focalidad tumoral específico no estuvo asociada con la presencia de TLC en el espécimen quirúrgico (OR 0.976 , IC95\% 0.444-2.143). Esta asociación no fue estadísticamente significativa, con un valor de $\mathrm{p}=$ 0.951 .

Se encontró una frecuencia de compromiso ganglionar correspondiente al $27.1 \%$. La prevalencia del compromiso ganglionar fue estadísticamente homogénea en el grupo de pacientes con TLC y sin TLC (30.9\% vs. $25.9 \%$, RP 1.20, IC95\% 0.69-2.12). La probabilidad de identificar compromiso ganglionar no estuvo asociada con la presencia de TLC en el espécimen quirúrgico (OR 1.281, IC95\% 0.600-2.736). Esta asociación no fue estadísticamente significativa, con un valor de $\mathrm{p}=$ 0.522 .

\section{DISCUSIÓN}

La relación entre TLC y CPT fue propuesta inicialmente en 1955 por Dailey (8). Desde entonces, se han reportado estudios con esta asociación, tanto a favor como en contra, a nivel genético e histopatológico.
Un metaanálisis de 71 estudios retrospectivos, sugirió que el CPT mostró a características clinicopatológicas favorables en el grupo de especímenes con TLC, con una incidencia reducida de extensión tumoral extratiroidea, metástasis a los ganglios linfáticos cervicales y metástasis a distancia, a diferencia de los pacientes sin TLC (9). Sin embargo, a diferencia de las características anatomopatológicas, la multifocalidad se correlacionó positivamente con el hallazgo de TLC. La multifocalidad se ha considerado como una característica asociada con el desarrollo de CPT, y no con su agravación, por tanto, estos hallazgos fueron congruentes con estudios previos que informaron un papel protector de TLC en la progresión de CPT (18). En nuestro estudio de investigación, la prevalencia de las características anatomopatológicas pronósticas para el CPT fue estadísticamente similar, no encontrándose asociación entre la TLC y las variables del estudio, sin embargo, cuantitativamente se encontró una mayor frecuencia de multifocalidad en pacientes con TLC.

En cuanto al tamaño tumoral, en este estudio no hubo diferencias estadísticamente significativas entre la presencia o ausencia de TLC, hallazgos que se correlacionan con la literatura (19). Algunos estudios han sugerido un curso menos agresivo del CPT con LPT (20), con tendencia a un menor tamaño tumoral, menor compromiso de ganglios linfáticos y mayor sobrevida, sin embargo, tampoco hubo diferencias significativas en el presente estudio en estas variables.

Aunque el mecanismo por el cual la TLC afecta al CPT no está claro, se han sugerido varias hipótesis. Entre ellas, el carcinoma inducido por inflamación se ha propuesto como uno de los posibles mecanismos. La respuesta inflamatoria activada en la TLC, que implica la producción de mediadores por parte de las células inmunitarias en un estado de inflamación crónica, podría crear un entorno favorable para la transformación maligna en la glándula tiroides (21). Sin embargo, esta hipótesis tiene dificultades para explicar el papel protector de la TLC contra la progresión del CPT. 
En este contexto, la autoinmunidad inducida por la defensa tumoral, como las células $\mathrm{T}$ citotóxicas específicas de la tiroides, podría jugar un papel. La tiroglobulina y la peroxidasa tiroidea, que se presentan en las células presentadoras de antígenos y las células foliculares, son los principales antígenos diana de las reacciones inmunitarias celulares, y estas reacciones inmunitarias pueden dar lugar a una destrucción específica de la glándula tiroides específica del objetivo (22).

Se propuso una predisposición genética como otro posible mecanismo de las propiedades protectoras de la TLC contra la progresión de la CPT, porque la mutación BRAF V600E, un marcador de comportamiento más agresivo en la CPT se detectó con menos frecuencia en pacientes con TLC coexistente que en aquellos sin TLC (23).

En conclusión, pese a que existen estudios que demuestran claramente que entre los pacientes con CPT, la TLC coexistente se asocia con mejores características anatomopatológicas, nuestros resultados no respaldaron dicha hipótesis. Por el momento, se considera que el tratamiento en estos casos debe consistir en una tiroidectomía total, ya que se ha documentado mayor prevalencia de multifocalidad en los casos de CPT con TLC. Aunque el mecanismo subyacente no está claro, los hallazgos de otros estudios podrían utilizarse para predecir el pronóstico de CPT en entornos clínicos. Se requerían de prospectivos en pacientes con TLC para determinar la hipótesis planteada en estudios retrospectivos.

En nuestro estudio de investigación las características anatomopatológicas del CPT fueron similares en el grupo de especímenes con y sin TLC. Debido a la retrospectividad del presente estudio, se recomienda la realización de estudios prospectivos de pacientes en los cuales se definan las características anatomopatológicas del CPT y los hallazgos compatibles con TLC con criterios actualizados y universales, y para el último caso, con la confirmación serológica de la enfermedad.
CONFLICTOS DE INTERESES: Los autores no declaran conflictos de interés.

FINANCIACION: El trabajo de investigación fue financiado por la Vicerrectoría de Investigación de la Universidad de Cartagena. Se desarrolló en el marco del Plan de Fortalecimiento del Grupo de Investigación Scalpellum. Acta de Compromiso No. 042. Resolución No. 833 de 2018.

\section{REFERENCIAS BIBLIOGRÁFICAS}

1. Huber G, Staub JJ, Meier C, Mitrache C, Guglielmetti M, Huber P, Braverman LE. Prospective study of the espontaneous course of subclinical hypothyroidism: prognostic value of thyrotropin, thyroid reserve, and thyroid antibodies. J Clin Endocrinol Metab. 2002; 87(7):3221-6.

2. Zaletel K, Gaberscek S. Hashimoto's thyroiditis: From genes to the disease. Curr Genomics. 2011; 12(8):57688.

3. Hashimoto H. Zur kenntniss der lymphömatosen verändererung der schilddrüse. Archiv für Klinishe Chirugie. 1912; 97:219-48.

4. Siegel RL, Miller KD, Jemal A. Cancer statistics, 2018. CA Cancer J Clin. 2018; 68(1):7-30.

5. Surveillance, Epidemiology, and End Results (SEER) Program. Stat Database: Mortality-All COD, Aggregated Whit State, Total US (1969-2015). Bethesda, MD: National Cancer Institute, Division of Cancer Control and Population Sciences, Surveillance Research Program, Cancer Statistics Branch; 2015; underlying mortality data provided by National Center for Health Statistics 2014.

6. Siegel RL, Miller KD, Jemal A. Cancer statistics, 2018. CA Cancer J Clin. 2018; 68(1):7-30.

7. Lindsay S, Dailey ME, Friedlander J, Yee G, Soley MG. Chronic thyroiditis: a clinical and pathologic study of 354 patients. J Clin Endocrinol Metab. 1952; 12(12):15781600.

8. Dailey ME, Lindsay S, Skahen R. Relation of thyroid neoplasms to Hashimoto disease of the thyroid gland. AMA Arch Surg. 1955; 70(2):291-7.

9. Moon S, Chung HS, Yu JM, Yoo HJ, Park JH, Kim DS, Park YJ. Associations between Hashimoto Thyroiditis 
and Clinical Outcomes of Papillary Thyroid Cancer: A Meta-Analysis of Observational Studies. Endocrinol Metab (Seoul). 2018; 33(4):473-484.

10. Hernández B, Velasco HE. Encuestas Transversales. En: Hernández M. Epidemiología. Diseño y análisis de estudios. México: Editorial Médica Panamericana. p 191-211.

11. Lloyd RV, Osumura RY, Klöpple G, Rosai J. World Health Organization \& International Agency for Research on Cancer. 2017 WHO Classification of Tumours of Endocrine Organs.

12. Nosé V. Diagnostic Pathology: Endocrine. 2 ed. Canadá: Elsevier, 2018.

13. Hung YP, Barletta JA. A user's guide to noninvasive follicular thyroid neoplasm with papillary-like nuclear features (NIFTP). Histopathology. 2018; 72(1):5369.

14. Romero AE, Parra R, Chinchilla SI, De Los Reyes A, Llamas A, Diferencias y controversias entre el reporte de patología y la interpretación clínica en patología tiroidea. I parte: Factores pronósticos. Rev Colomb Cancerol. 2017; 21(3):160-65.

15. Castañeda JA, Vanegas H, Rodríguez MN. Presentación de datos estadísticos. En: Ruiz A, Morillo LE. Epidemiología clínica: Investigación clínica aplicada. Colombia: Editorial Medica Panamericana. p 487-507.

16. Hernández B, Velasco HE. Encuestas Transversales. En: Hernández M. Epidemiología. Diseño y análisis de estudios. México: Editorial Médica Panamericana. p 191-211.
17. Plackett RL. Karl Pearson and the Chi-Squared Test. Inter Statis Rev. 1983; 51(1):59-72.

18. Lee JH, Kim Y, Choi JW, Kim YS. The association between papillary thyroid carcinoma and histologically proven Hashimoto's thyroiditis: a meta-analysis. Eur J Endocrinol 2013; 168:343-9.

19. Kim SK, Song KH, Lim SD, Lim YC, Yoo YB, Kim JS, et al. Clinical and pathological features and the BRAF (V600E) mutation in patients with papillary thyroid carcinoma with and without concurrent Hashimoto thyroiditis. Thyroid. 2009; 19(2):137-41.

20. Ahn D, Heo SJ, Park JH, Kim JH, Sohn JH, Park JY, et al. Clinical relationship between Hashimoto's thyroiditis and papillary thyroid cancer. Acta Oncol. 2011; $50(8): 1228-34$.

21. Liotti F, Visciano C, Melillo RM. Inflammation in thyroid oncogenesis. Am J Cancer Res 2012; 2: 286-97.

22. Ehlers M, Schott M. Hashimoto's thyroiditis and papillary thyroid cancer: are they immunologically linked? Trends Endocrinol Metab 2014; 25:656-64.

23. Zhang Q, Liu SZ, Zhang Q, Guan YX, Chen QJ, Zhu QY. Meta-analyses of association between BRAF (V600E) mutation and clinicopathological features of papillary thyroid carcinoma. Cell Physiol Biochem 2016; 38:763-76. 